\section{UK national survey of prophylactic YAG iridotomy}

HG Sheth'1, R Goel ${ }^{2}$ and S Jain ${ }^{2}$

\begin{abstract}
Purpose Acute angle-closure glaucoma is a common ophthalmic emergency and individuals with shallow anterior chambers and suspected narrow angles are increasingly referred to the hospital eye service for assessment. There appears to be variation in subsequent management, with no national consensus or college guidelines. This study ascertains the current use of prophylactic YAG iridotomy in patients with no known history of an acute angle-closure glaucoma attack, and also the methods used in patient selection. Materials and methods Questionnaire-based survey mailed to 650 UK consultant ophthalmologists with a covering letter in 2003.
\end{abstract}

Results A total of 546 questionnaires were returned. In all, 408 respondents $(74.7 \%)$ confirmed they perform prophylactic YAG iridotomy and of these $347(85.0 \%)$ use patient symptoms and $268(65.6 \%)$ presenting IOP in patient selection, $394(96.6 \%)$ perform gonioscopy and $97(23.8 \%)$ use some form of provocative test first. A total of 135 (25.3\%) stated they do not perform this procedure. Conclusion This study reveals current national practice among UK ophthalmologists, with variations in the assessment of patients with narrow angles but a high uptake of prophylactic YAG iridotomy.

Eye (2005) 19, 981-984. doi:10.1038/sj.eye.6701696; published online 17 September 2004

Keywords: glaucoma; narrow angle; prophylactic; Nd:YAG laser iridotomy

\section{Introduction}

Ophthalmologists in the UK are increasingly required to appropriately manage individuals with shallow anterior chambers and suspected narrow angles but no symptoms or past ophthalmic history, who have been referred to the hospital eye service by their optometrist for assessment and consideration for prophylactic Nd:YAG laser peripheral iridotomy (YPI).

The prevalence of glaucoma varies with age and ethnic origin and for middle-aged Caucasians is reported to be $2.1-3.0 \%$ for the open-angle form and $0.3 \%$ for angle-closure. ${ }^{1,2}$ Worldwide, however, angle-closure glaucoma is the leading cause of blindness. ${ }^{3}$ Narrow angles are an anatomical trait, which predispose to two potential sight-threatening conditions; firstly, an acute angle-closure episode, which presents relatively commonly in the Western world as an ophthalmic emergency, and secondly, a more chronic angle-closure glaucoma (PACG) more common among Oriental and Eskimo populations. ${ }^{3}$ A previous series has demonstrated the characteristics of eyes that develop angle-closure; namely shallow anterior chambers, narrow angles, small corneal diameter, small corneal radius of curvature, short axial length, thick lens, and relative anterior displacement of the lens. ${ }^{4}$

There is variation in the estimated prevalence of narrow or 'occludable' angles across the globe, quoted as 2.2-5.0\% in Caucasian subjects over 55 years of age, rising markedly to $47.8 \%$ in similarly aged Vietnamese subjects. ${ }^{5-7}$ The annual incidence of acute angle-closure glaucoma (AACG) is estimated at 3.8 per 100000 in unselected European populations. ${ }^{8}$ Wilensky et al followed a cohort of 129 predominantly Caucasian individuals with narrow angles or anterior chamber depth less than $2 \mathrm{~mm}$ and no symptoms for a mean of 2.7 years. Eight patients $(6.2 \%)$ developed an acute angle-closure attack with symptoms during the follow-up period and an additional 17 patients (13.2\%) were found to have angles closed with synechiae or by apposition on follow-up gonioscopy. ${ }^{9}$ Recently, Thomas et al have shown, in an Asian cohort, that $22 \%$ of primary angle closure suspects developed raised intraocular pressure (IOP) or synechiae over a 5-year period $^{10}$ and in a separate smaller group with
${ }^{1}$ Department of Ophthalmology St Thomas' Hospital London, UK

${ }^{2}$ Department of Ophthalmology Queen Mary's Hospital Sidcup, UK

Correspondence: HG Sheth Department of Ophthalmology

St Thomas' Hospital Lambeth Palace Road London SE1 7EH, UK Tel/fax: + 442071887188 E-mail: drhitensheth@ yahoo.co.uk

Received: 8 May 2004 Accepted: 13 July 2004 Published online:

17 September 2004

The authors received no funding for this work No conflict of interest 
known primary angle closure $28.5 \%$ progressed to glaucoma as evidenced by disc damage and perimetry defects over the same period. ${ }^{11}$ Despite these studies, there is still paucity of larger studies with long-term data on the natural history of treated and untreated narrow angles, particularly among Caucasian populations.

An AACG episode is associated with significant morbidity but a relatively safe, simple and noninvasive prophylactic procedure in the form of YPI is available. Complications of this technique are uncommon and usually transient, but include bleeding from the iridotomy site, acutely raised IOP, anterior uveitis, posterior synechiae formation, acceleration of cataract development, and corneal decompensation. ${ }^{12}$ Additionally, subjective monocular blurring or a bluish hue have been reported ${ }^{13}$ and are thought to be related to suboptimal siting of the YPI.

In the 1950s, Lowe ${ }^{4}$ documented higher rates of acute angle-closure attacks in the untreated fellow eyes of patients with previous AACG and now few clinicians would deny such a patient an iridotomy in the fellow eye. The situation is less clear in those who present incidentally with shallow anterior chambers or narrow angles and while prompting recent debate ${ }^{14}$ there remains no general consensus on the role of YPI in asymptomatic (no pain, redness, or visual symptoms such as blurring or haloes, and no documented history of AACG) individuals with potential for occlusion.

Anecdotal evidence suggests that clinical practice varies widely within units, locally and nationally. This study was therefore conducted to determine the preferences and current practice patterns of UK consultant ophthalmologists in an attempt to assist all ophthalmologists in their decision to proceed to iridotomy or not.

\section{Methods}

A five-point questionnaire was mailed in May 2003 to all UK ophthalmologists appearing on our database of 650 , together with an explanatory letter. It asked 'do you perform YAG iridotomy for shallow anterior chambers and/or narrow angles prophylactically in patients with no known history of an acute angle-closure episode?' It then asked if patient symptoms, IOP, gonioscopy, and provocative tests were employed in patient selection. Respondents were required to circle a yes/no option for each question, to provide further information on any provocative tests used and finally whether glaucoma was the respondents' subspecialty/area of interest. Data were collated anonymously and analysed.

\section{Results}

In all, 546 consultant ophthalmologists returned their questionnaire (84\% response rate), 543 correctly completed and three incomplete. A total of 135 respondents $(25.3 \%)$ indicated they never perform prophylactic YPI and 14 (10.4\%) of this group quoted glaucoma as their subspecialty or area of interest.

A total of 408 consultant ophthalmologists (74.7\%) confirmed they perform prophylactic YPI. Of these, 347 $(85.0 \%)$ take into account patient symptoms/history and $268(65.6 \%)$ presenting IOP. In all, $394(96.6 \%)$ confirmed gonioscopy is essential in patient selection, and within this group 234 expressed a preference for Goldmann only, 120 for Zeiss 4 mirror only and 40 for consecutive use of both techniques. Nine $(2.2 \%)$ reported that they routinely attempt formal manual measurement of AC depth (Table 1).

Table 1

\begin{tabular}{|c|c|c|c|c|}
\hline Questionnaires sent & 650 & & & \\
\hline Responses & 546 & Yes 408 & No 135 & Incomplete 3 \\
\hline \multicolumn{5}{|c|}{ Criteria used in patient selection } \\
\hline Symptoms & 347 & \multirow{4}{*}{ Goldmann 234} & \multirow{4}{*}{ Zeiss 120} & \multirow{4}{*}{ Both 40} \\
\hline IOP & 268 & & & \\
\hline Gonio & 394 & & & \\
\hline AC depth & 9 & & & \\
\hline \multicolumn{5}{|l|}{ Provocative tests } \\
\hline \multirow[t]{4}{*}{ Drops } & 79 & Tropicamide 26 & & \\
\hline & & Phenylephrine 13 & & \\
\hline & & both 6 & & \\
\hline & & Cyclopentolate 2 & & \\
\hline Dark prone test & 15 & & & \\
\hline DPT and water & 3 & & & \\
\hline
\end{tabular}


A total of 97 (23.8\%) indicated that they use a provocative test. In all, $79(19.4 \%)$ use topical agents and of these 26 stated a preference for tropicamide 0.5 or $1 \%$ only, 13 phenylephrine 2.5 or $10 \%$ only, six both agents and two cyclopentolate $1 \%$. Four respondents $(0.98 \%)$ use pilocarpine only in an attempt to induce an IOP reduction. In all, $15(3.7 \%)$ use the dark room/prone test with three more admitting to additional fluid loading. Of the above 366 interventionalists, $113(27.7 \%)$ are glaucoma specialists. Several respondents stated they looked forward to our results to act as an informal form of guidance.

\section{Discussion}

This survey reveals that the majority of consultant ophthalmologists in the UK $(74.7 \%)$ do perform prophylactic YPI in eyes with narrow angles but no history of an AACG attack and, with a response rate of $84 \%$, it may be accepted as broadly representative.

The aim of YPI is to spare the patient from the potentially devastating symptoms and visual morbidity associated with an acute angle-closure attack. Two recent prospective studies ${ }^{15,16}$ confirm YPI to be as effective as surgical iridectomy in the treatment of fellow eyes, YPI being noninvasive and therefore the preferred mode of prophylaxis. Some may regard the above as overtreatment citing inappropriate utilisation of resources, theoretical YAG laser risks as listed above, an absence of long-term followup data, and a slight residual risk of angle-closure and elevation of IOP even in the presence of a patent iridotomy.

Four respondents appeared to suggest that they perform YPI on the basis of patient symptoms or history alone. Nine reported they routinely attempt formal measurement of anterior chamber depth/angle width during slit-lamp examination, all preferring Van Herrick's method $^{17}$ in which limbal anterior chamber depth is expressed as a fraction of corneal thickness. None reported any attempt at objective measurement of axial anterior chamber depth, for example, A-scan ultrasound.

With regard to provocative tests, ${ }^{18}$ instillation of drops is common practice compared to the dark room prone test and personal preference appears to be exercised in choosing the type and strength of pharmacological agent. The majority of 'drop users' use tropicamide only, some use a combination of pilocarpine and phenylephrine, previously described as the Mapstone test, ${ }^{19}$ and a minority cyclopentolate. All re-check subsequent IOP with a rise of $4-10 \mathrm{mmHg}$ generally being deemed significant. A few additionally use pilocarpine, stating they look for an IOP fall of $>8 \mathrm{mmHg}$ as an indication for proceeding to YPI. Notably, among respondents specialising in glaucoma, extremes of practice were identified ranging from gonioscopy only to a repertoire including indentation gonioscopy, dark room/prone test, and then mydriatics.
Additionally, there is a group who did not use elective provocative tests but who will perform prophylactic YPI if, after routine mydriasis, the patient complains of symptoms or an elevation in IOP is documented. Provocative tests have been used for over 30 years in the UK but the dark room prone test is not in contemporary use. Possible reasons include pressures on clinic time, patient limitations such as frailty or poor mobility, clinician apathy, and a shift in organisational culture with an increasingly low threshold to proceed to YPI. Certainly, previous authors have questioned the value of such tests, suggesting that they have no increased predictive value than a slit-lamp examination combined with gonioscopy. ${ }^{9,20,21}$

The potential for occludability under natural circumstances is only one factor in deciding whether to proceed to YPI. Many respondents advocated YPI in eyes requiring repeated mydriasis for diagnosis or treatment of, for example, diabetic retinopathy and the Rotterdam study ${ }^{5}$ estimates the incidence of AACG under such circumstances at 3.8 per 10000 while another study, also in a Caucasian population, suggests up to $15 \%$ of AACG cases could be considered as drug induced. ${ }^{8}$ Several respondents also indicated a lower threshold for YPI in patients with pigment dispersion syndrome and those who regularly travel to countries with limited specialist healthcare.

Miscellaneous comments regarding alternative anglewidening options included a preference for lens extraction and implant if significant cataract is present, ${ }^{22}$ surgical iridectomy over YAG laser iridotomy, diode laser iridoplasty, and simple clinic follow-up. The use of topical pilocarpine to decrease the likelihood of AACG did not appear to be current common practice, and it should not be relied upon for prevention or treatment. Several respondents who stated they never perform prophylactic YPI did however state that they would refer such patients to colleagues specialising in anterior segment/glaucoma for a final opinion.

In view of the lack of widely accepted and published definitions of angle-closure glaucoma and a degree of subjectivity in assessing the narrow angle, our questionnaire was kept necessarily simple. The exact wording and content of the questions (as detailed in methods) may have led to certain interpretations that inevitably had an impact on respondents' answers and, in turn, the results of the survey. Distinct entities exist under the umbrella of 'narrow' or 'angle-closure glaucomas' and terms in the literature are often inconsistent, arbitrary, and evolving, such that accurate comparison of data across studies becomes difficult. ${ }^{23}$ Foster et $a^{24}$ have recently highlighted deficiencies in the epidemiological definition of the narrow or occludable angle and the implications in an east Asian population and a practical definition for readers is any eye in which $180^{\circ}$ (possibly $270^{\circ}$ ) or more of the angle is not visible on 
gonioscopy. Correctly speaking, primary angle-closure glaucoma (PACG) requires the presence of optic disc change and/or a reproducible defect on perimetry, while a narrow angle with raised IOP but no optic disc damage should be termed angle-closure glaucoma suspect.

Ophthalmologists in Europe and the USA are familiar with the dramatic acute form of angle-closure of which individuals with narrow angles have an increased but not absolute risk. Our study relates to the prophylactic use of YPI in this group with the aim of preventing pupil block, an acute rise in IOP and associated visual morbidity. As previously stated, the prevalence of occludable angles in Caucasians is $2.2-5.0 \%$ but only a small proportion will go on to develop raised IOP and optic neuropathy, that is true PACG that has a prevalence of $0.09-0.17 \%$. While these numbers may seem low, it should be remembered that the optometry service is particularly well established in the UK and there is a culture among the majority of the population to attend a routine eye test. Taking the UK population as 80 million and the above prevalence rates, even with conservative projections, thousands of individuals in the UK may eventually reach the hospital eye service for consideration for YPI.

In summary, this novel study presents the current practice of the majority of UK consultant ophthalmologists with 74.7\% performing YPI as prophylaxis against AACG in patients who have narrow/occludable angles but have not sustained an acute attack in either eye. The most popular regime applied in patient selection for YPI is a combination of symptoms, presenting IOP and Goldmann indirect gonioscopy. Our survey yields new data in a clinical area where there is a deficient evidence base and will hopefully encourage further discussion in the UK to firstly adopt a precise and practical definition of the occludable angle and also to clarify the role and limitations of prophylactic YPI.

\section{Acknowledgements}

We thank Charles Hugkulstone for his encouragement, Mrs Julia Russell for administrative assistance and Vik Sharma for initial help. We also thank all respondents.

\section{References}

1 Klein BE, Klein R, Sponsel WE, Franke T, Cantor LB, Martone J et al. Prevalence of glaucoma. The Beaver dam Eye Study. Ophthalmology 1992; 99(10): 1499-1504.

2 Mitchell P, Smith W, Attebo K, Healey PR. Prevalence of open-angle glaucoma in Australia. The Blue Mountains Eye Study. Ophthalmology 1996; 103(10): 1661-1669.

3 Foster PJ, Johnson GJ. Glaucoma in China-how big is the problem? Br J Ophthalmol 2001; 85: 1277-1282.

4 Lowe RF. The second eye: an analysis of 200 cases. $\mathrm{Br}$ J Ophthalmol 1962; 45: 641-650.

5 Wolfs RC, Grobbee DE, Hofman A, de Jong PT. Risk of acute angle-closure glaucoma after diagnostic mydriasis in nonselected subjects: the Rotterdam Study. Invest Ophthalmol Vis Sci 1997; 38: 2683-2687.

6 Leibowitz HM, Kreuger DR, Maunder LR, Milton RC, Kini MM, Kahn HA et al. The Framingham study monograph. Surv Ophthalmol 1980; 24: 335-610.

7 Nguyen N, Mora JS, Gaffney MM, Ma AS, Wong PC, Iwach AG et al. A high prevalence of occludable angles in a Vietnamese population. Ophthalmology 1996; 103: 1426-1431.

8 Vadot E, Grateau C. The frequency of acute glaucoma crises. Implications for the detection of the risk of closure. Bull Soc Ophthalmol Fr 1989; 89: 675-677.

9 Wilensky JT, Kaufman PL, Frohlichstein D, Gieser DK, Kass $\mathrm{MA}$, Ritch $\mathrm{R}$ et al. Follow-up of angle-closure glaucoma suspects. Am J Ophthalmol 1993; 115: 338-346.

10 Thomas R, George R, Parikh R, Muliyil J, Jacob A. Five year progression of primary angle closure suspects to primary angle closure: a population based study. $\mathrm{Br} J$ Ophthalmol 2003; 87: 450-454.

11 Thomas R, Parikh R, Muliyil J, Kumar RS. Five year progression of primary angle closure to primary angle closure glaucoma: a population based study. Acta Ophthalmol Scand 2003; 81: 480-485.

12 Maraffa M, Marchini G, Pagliarusco A, Perfetti S, Toscano A, Brunelli C et al. Corneal endothelium in Nd:YAG-laser iridotomy. Ophthalmic Surg Lasers 1995; 26(6): 519-523.

13 Murphy PH, Trope GE. Monocular blurring - a complication of YAG laser iridotomy. Ophthalmology 1991; 98(10): 1539-1542.

14 Slamovits T, Dutton J. Should patients with anatomically narrow angles have prophylactic iridectomy? Surv Ophthalmol 1996; 41: 31-36.

15 Fleck BW, Wright E. A randomised prospective comparison of operative PI and YAG PI treatment of acute angle closure glaucoma: 3-year visual acuity and intraocular pressure outcome. Br J Ophthalmol 1997; 81: 884-888.

16 Schwenn O, Sell F. Prophylactic Nd:YAG-laser iridotomy versus surgical iridectomy: a randomised, prospective study. Ger J Ophthalmol 1995; 4: 374-379.

17 Van Herrick W, Shaffer RN. Estimation of width of angle of anterior chamber: incidence and significance of the narrow angle. Am J Ophthalmol 1969; 68: 626.

18 Wand M. Provocative tests in angle-closure: a review with commentary. Ophthalmic Surg 1974; 5: 32-37.

19 Mapstone R. Outflow changes in positive provocative tests. Br J Ophthalmol 1977; 61: 634-636.

20 McGalliard JN, Wishart PK. The effect of Nd:YAG iridotomy on intraocular pressure in hypertensive eyes with shallow anterior chambers. Eye 1990; 4: 823-829.

21 Wishart PK. Can the pilocarpine phenylephrine provocative test be used to detect covert angle closure? $\mathrm{Br}$ J Ophthalmol 1991; 75: 615-618.

22 Pereira FA, Cronemberger S. Ultrasound biomicroscopic study of anterior segment changes after phacoemulsification and foldable IOL implantation. Ophthalmology 2003; 110(9): 1799-1806.

23 Foster PJ, Buhrmann R, Quigley HA, Johnson GJ. The Diagnosis and classification of glaucoma in prevalence surveys. Br J Ophthalmol 2002; 86: 238-242.

24 Foster PJ, Aung T, Nolan WP, Machin D, Baasanhu J, Khaw PT et al. Defining 'occludable' angles in population surveys: drainage angle width, peripheral anterior synechiae and glaucomatous optic neuropathy in east Asian people. Br J Ophthalmol 2004; 88: 486-490. 